\title{
Possible local anesthetic resistance in Emery-Dreifuss muscular dystrophy during regional anesthesia
}

\author{
Alexander J Kim ${ }^{1}$, Thomas M Halaszynski ${ }^{2}$, and Lori-Ann Oliver ${ }^{2}$ \\ Department of Anesthesiology, Perioperative and Pain Medicine, ${ }^{1}$ Brigham and Women's Hospital, Harvard \\ Medical School, Boston, MA, ${ }^{2}$ Yale School of Medicine, New Haven, CT, USA
}

We recently provided perioperative anesthetic care for a 31-year-old man with Emery-Dreifuss muscular dystrophy (EDMD) presenting for bilateral Achilles lengthening and equinoplanovalgus deformity correction. We performed bilateral popliteal sciatic and femoral nerve blockade, using ultrasound guidance and nerve stimulator confirmation, mainly to avoid some potential pitfalls of general anesthesia and airway instrumentation. However, the patient appeared resistant to the effects of local anesthetic. The few published reports of anesthesia management for such patients describe general and neuraxial techniques [1,2]. To our knowledge, this is the first written description of regional anesthesia in this rare patient population.

EDMD is a rare $\mathrm{X}$-linked condition that primarily affects skeletal muscle, leading to joint contractures with progressive weakness, and cardiac muscle, leading to malignant arrhythmias and cardiomyopathy [3]. EDMD presents unique challenges to the anesthetist, including arrhythmias (i.e., atrioventricular heart block), cardiomyopathy, possible compromised airway mechanics leading to difficult intubation (i.e., cervical paraspinal contractures and hypoplasia of the third to fifth cervical vertebral bodies that can influence neck flexion and extension),

Corresponding author: Alexander J Kim, M.D.

Department of Anesthesiology, Perioperative and Pain Medicine, Brigham and Women's Hospital, Harvard Medical School, 75 Francis Street, Boston, MA 02115, USA

Tel: 1-617-732-9060, Fax: 1-617-732-9050

Email: akim30@bwh.harvard.edu

ORCID: https://orcid.org/0000-0002-5645-1877

Received: March 6, 2017.

Accepted: March 12, 2017.

Korean J Anesthesiol 2017 August 70(4): 477-478

https://doi.org/10.4097/kjae.2017.70.4.477 potential for difficult neuraxial access due to paraspinal muscle contractures, and concern for adverse medication reactions (i.e., succinylcholine exaggerated hyperkalemic response or risk of rhabdomyolysis [3] and negative volatile anesthetic reactions [1]). Considering the challenges of anesthetic management in this patient, it seemed reasonable that bilateral sciatic and femoral nerve blockade could avoid airway instrumentation and general anesthesia. Notably, however, the patient commented preoperatively that “...every time I received a local anesthetic injection at the dentist's office, I required multiple injections in order to feel any effect."

A popliteal approach nerve block of the sciatic nerves was performed bilaterally with ultrasound, visualizing the circumferential perineural spread of ropivacaine at the junction of the tibial and common peroneal nerves. Bilateral perineural catheters were then placed. Then, a single-shot block of the femoral nerves at the inguinal crease was performed bilaterally with ultrasound, visualizing the circumferential perineural spread of ropivacaine. Despite nerve stimulation confirmation (Stimuplex ${ }^{\circledR}$ HNS12, B. Braun Medical, Bethlehem, PA, USA) with less than 0.5 milliamperes and ultrasound imaging of perineural local anesthetic spread at all block sites (Fig. 1), the patient reported intact sensation to temperature (alcohol pad testing) and nociception (blunt needle prick) within all affected nerve distributions. Both sciatic catheters were then bolused with lidocaine, yet the patient reported achieving only a minimal degree of temperature sensation change. Owing to inadequate surgical anesthesia with the regional technique, we decided to administer total intravenous anesthesia (TIVA) using a laryngeal mask airway, thereby avoiding endotracheal intubation, muscle relaxation, and volatile anesthetics. The surgical procedure was completed within 2 hours with evidence of stable hemodynamics with TIVA using a propofol infusion and intermittent boluses of

(c) This is an open-access article distributed under the terms of the Creative Commons Attribution Non-Commercial License (http://creativecommons.org/ licenses/by-nc/4.0/), which permits unrestricted non-commercial use, distribution, and reproduction in any medium, provided the original work is properly cited. 
A

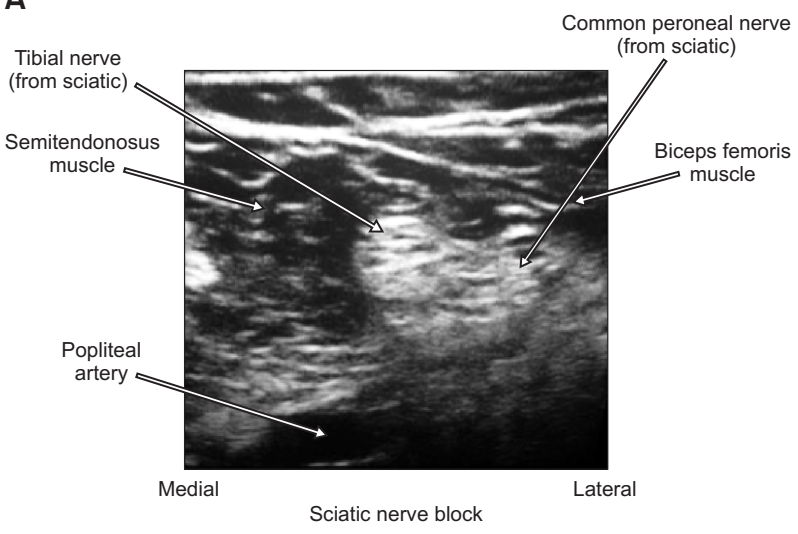

B

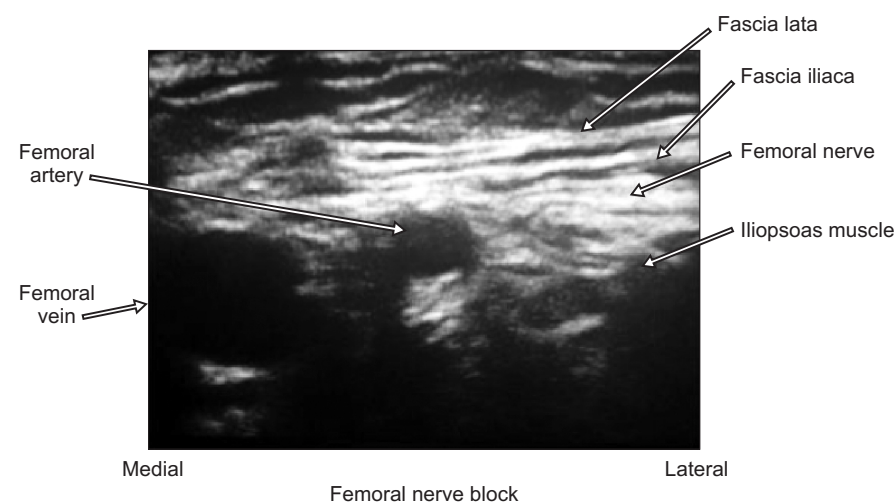

Fig. 1. (A) Ultrasound imaging of the tibial and common peroneal branches of the sciatic nerve and surrounding structures. (B) Ultrasound imaging of the femoral nerve and surrounding structures.

hydromorphone.

The patient had atypical requirements for local anesthetics (testing upper limit guidelines), while clinically achieving minimal efficacy with peripheral nerve blocks. Guidelines for the recommended local anesthetic maximum are based on intravenous administration (i.e., ropivacaine $3 \mathrm{mg} / \mathrm{kg}$ and lidocaine $4-5$ $\mathrm{mg} / \mathrm{kg}$ ) and anesthesia providers often extrapolate these same local anesthetic limits when performing regional techniques. Resistance to local anesthetic effects has rarely been reported, with only one report of decreased efficacy and duration of topical and intradermal local anesthetic administration in patients with Ehler-Danlos syndrome (it was postulated that this was due to the laxity and density of abnormal connective tissues [4], which is clearly not related to muscular dystrophy). There is also research on specific sodium channel variants that may be linked to resistance to local anesthetics [5]. We acknowledge that there are many points to confirm before declaring that our patient clearly demonstrated resistance to local anesthetic. However, he expressed interest in genetic testing of his sodium channels to look for abnormalities related to local anesthetic resistance. If an atypical sodium channel variant were discovered, it could provide valuable insight toward determining if such a variant had a higher incidence in those with muscular dystrophy.

\section{References}

1. Aldwinckle RJ, Carr AS. The anesthetic management of a patient with Emery-Dreifuss muscular dystrophy for orthopedic surgery. Can J Anaesth 2002; 49: 467-70.

2. Kim OM, Elliott D. Elective caesarean section for a woman with Emery-Dreifuss muscular dystrophy. Anaesth Intensive Care 2010; $38: 744-7$.

3. Emery AE. Emery-Dreifuss muscular dystrophy and other related disorders. Br Med Bull 1989; 45: 772-87.

4. Arendt-Nielsen L, Kaalund S, Bjerring P, Høgsaa B. Insufficient effect of local analgesics in Ehlers Danlos type III patients (connective tissue disorder). Acta Anaesthesiol Scand 1990; 34: 358-61.

5. Dib-Hajj SD, Waxman SG. Diversity of composition and function of sodium channels in peripheral sensory neurons. Pain 2015; 156 : 2406-7. 